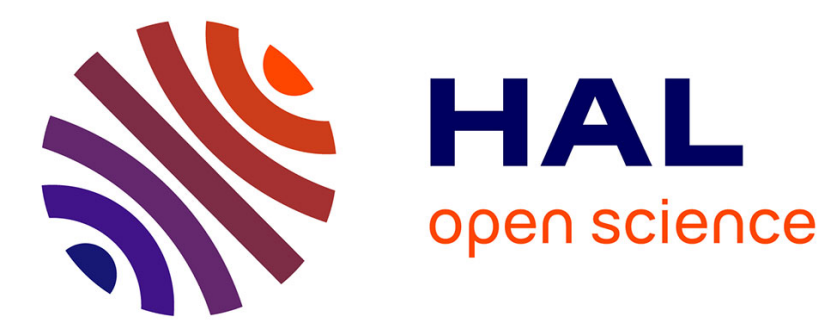

\title{
L'utilisation des mesures intensimétriques en acoustique des salles
}

\author{
J.-G. Migneron, P. Lemieux, P. Coté
}

\section{To cite this version:}

J.-G. Migneron, P. Lemieux, P. Coté. L'utilisation des mesures intensimétriques en acoustique des salles. Journal de Physique IV Proceedings, 1994, 04 (C5), pp.C5-151-C5-154. 10.1051/jp4:1994527 . jpa-00252960

\section{HAL Id: jpa-00252960 https://hal.science/jpa-00252960}

Submitted on 1 Jan 1994

HAL is a multi-disciplinary open access archive for the deposit and dissemination of scientific research documents, whether they are published or not. The documents may come from teaching and research institutions in France or abroad, or from public or private research centers.
L'archive ouverte pluridisciplinaire HAL, est destinée au dépôt et à la diffusion de documents scientifiques de niveau recherche, publiés ou non, émanant des établissements d'enseignement et de recherche français ou étrangers, des laboratoires publics ou privés. 


\title{
L'utilisation des mesures intensimétriques en acoustique des salles
}

\author{
J.-G. MIGNERON, P. LEMIEUX et P. COTÉ
}

\begin{abstract}
Laboratoire d'Acoustique, CRAD et Ecole d'Architecture, 1636 Félix-Antoine Savard, Université de Laval, Québec G1K 7P4, Canada
\end{abstract}

\begin{abstract}
The intensity measurement technics in architectural acoustics have been developed since the early 1980's. Its utilization has mainly been directed towards the solution of noise transmission or sound isolation problems to analyse the radiation of partition with or without opening and, in some more specialized absorption measurements. As far as the acoustics related to concert halls, the impulse response analysis continues to be the predominant measurement method, since the intensity measurement technics are not very compatible with the time analysis. However, different experiments of data gathering, recorded either in a tightly controlled directional mode or in a omnidirectional mode using a robot or a tridimensional probe, have been reported in concert halls and auditoriums. This paper reviews some of these experiments and presents more recent measurements made in concert halls, showing different technics of reflection analysis as well as a propagation summary at different points of location.
\end{abstract}

Les techniques intensimétriques ont été valorisées, depuis le début des années 80 pour leur utilisation dans le domaine de l'acoustique architecturale. Mais les principaux efforts ont porté surtout sur les problèmes de transmission ou d'isolation acoustique, afin d'analyser la radiation d'une paroi soumise au bruit, en présence ou non d'une ouverture, de même que, d'une façon plus spécialisée, sur la mesure des absorptions acoustiques $[1,2]$. Du côté de l'acoustique des salles, l'analyse de la réponse impulsionnelle a continué à prévaloir, les techniques intensimétriques étant difficilement compatibles avec l'analyse temporelle. Il faut cependant mentionner le récent travail de ABDOU et GUY qui, à l'aide d'un système d'acquisition rapide des six canaux d'une sonde intensimétrique tri-dimensionnelle, sont capables de produire à la fois les informations temporelles conventionnelles (RT, EDT, C80, STI, etc.) et un relevé polaire des premières lignes de flux [3].

Différentes expériences ont été mentionnées en matière d'acoustique des salles ou des locaux industriels, avec des acquisitions directionnelles contrôlées ou bien omni-directionnelles, par la robotique ou à l'aide d'une sonde multi-dimensionnelle $[4,5,6]$. Dans des conditions de laboratoire ou en acoustique des salles, il est possible d'utiliser des sources fixes, de radiation constante dans le temps (en intensité et en directivité), cette situation corrrespond à un champ acoustique stationnaire. C'est d'ailleurs dans ces conditions qu'un système robotique peut être utilisé le plus rapidement, en réduisant à son minimum le temps d'acquisition des niveaux d'intensité.

Il s'agit toujours d'exploiter le mieux possible les indications de directivité fournies par la nature vectorielle de l'intensité. En déterminant complètement (norme et direction) cette dernière, il est possible de localiser la provenance exacte du champ acoustique étudié et d'estimer simultanément sa contribution en énergie dans une direction donnée [7]. Quelques techniques, spécifiquement adaptées à l'acoustique architecturale, seront présentées pour l'analyse des réflexions et le bilan propagatif en différentes localisations d'une salle.

\section{TECHNIQUE DU BALAYAGE DES SURFACES RÉFLÉChISSANTES}

Cette première technique consiste à balayer, en visant avec la sonde intensimétrique, une surface réfléchissante déterminée. En prenant certaines précautions et en moyennant la mesure pendant un temps suffisamment long, on peut obtenir une distinction très significative de la contribution des différentes parois réfléchissantes d'une salle, tant en bandes de fréquence qu'en énergie.

Une application immédiate de cette technique concerne le réglage des réflecteurs mobiles, comme le montre la figure suivante, à propos des réflecteurs d'avant-scène de la salle Louis-Fréchette du Grand Thêâtre de Québec [8]. 


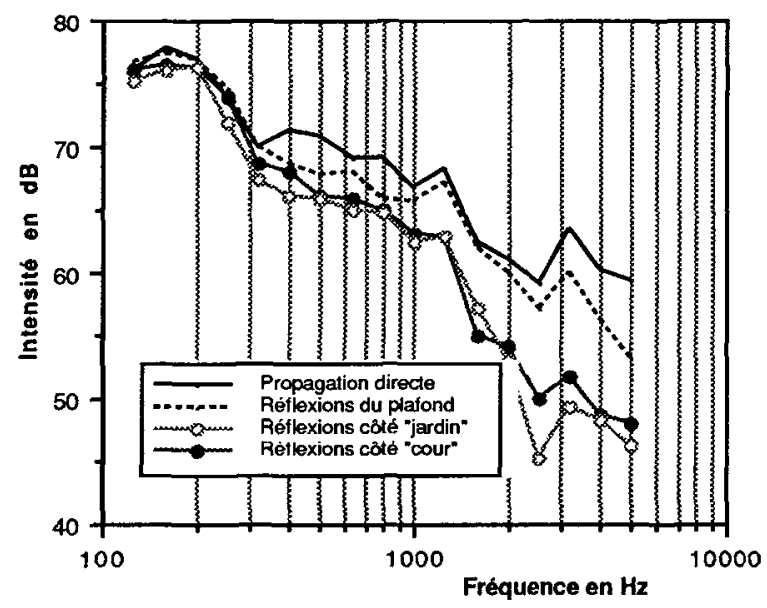

FIG. N¹: Exemple de distinction par balayage intensimétrique dès différents chemins de propagation dans une salle de concert.

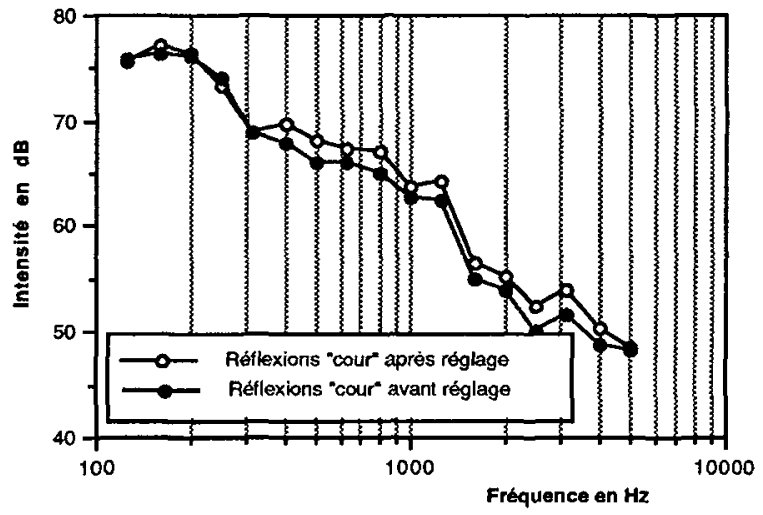

FIG. N22: Application au réglage des réflecteurs latéraux d'avant-scène.

\section{ACQuisition robotique de l'intensité propagative}

Le robot utilisé est construit autour de deux axes de rotation perpendiculaires entre eux, leur intersection étant placée juste au centre acoustique du doublet de microphones de la sonde. Les deux moteurs sont ramenés vers la base, afin d'agir comme contrepoids. Il peut être employé pour des relevés polaires automatiques de pas angulaire variable; le vecteur intensité composé étant toujours localisé dans un cône dont l'angle au sommet n'excède pas le pas angulaire de mesure. Les relevés polaires ont l'avantage de permettre d'interpréter convenablement tous les changements de signe qui affectent les mesures en champ diffus. Le temps moyen de déplacement entre deux positions de mesure est de $1 \mathrm{sec}$. et la précision de la position du centre acoustique de la sonde, dans toutes les directions de l'espace, de $1 \mathrm{~mm}$. D'autre part, le robot peut être utilisé pour la recherche automatique de la ligne de flux; on peut procéder ainsi, de façon itérative, à la détermination du vecteur résultant (une procédure automatique rapide a été programmée, elle utilise un pas angulaire de $5^{\circ}$ ) [9]. Un dispositif de discrimination spatial est actuellement à l'étude pour supprimer le plus possible les $\operatorname{composantes~en~} \cos \theta$.

\section{APPLICATION À LA DESCRIPTION DE L'ESPACE SONORE}

Les exemples qui suivent ont été relevés sur le parterre de la salle Albert Rousseau à Ste-Foy (1250 places deux niveaux de balcons) ainsi que dans l'auditorium du collège de Lévis (1100 places et un seul grand balcon), soit deux salles multifonctionnelles de la région de Québec utilisées pour la musique symphonique. Une source omni-directionnelle de forte puissance a été placée au centre de la conque d'orchestre et le robot intensimétrique déplacé en différente localisation du parterre. Dans tous les cas, l'axe des X reste parallèle à l'axe principal de la salle et orienté vers la scène. Le pas de déplacement 
angulaire généralement utlisé est de $30^{\circ}$ et la procédure automatique d'acquisition programmée pour l'hémisphère supérieur (le doublet de microphones étant placé à la hauteur de la tête d'un auditeur).

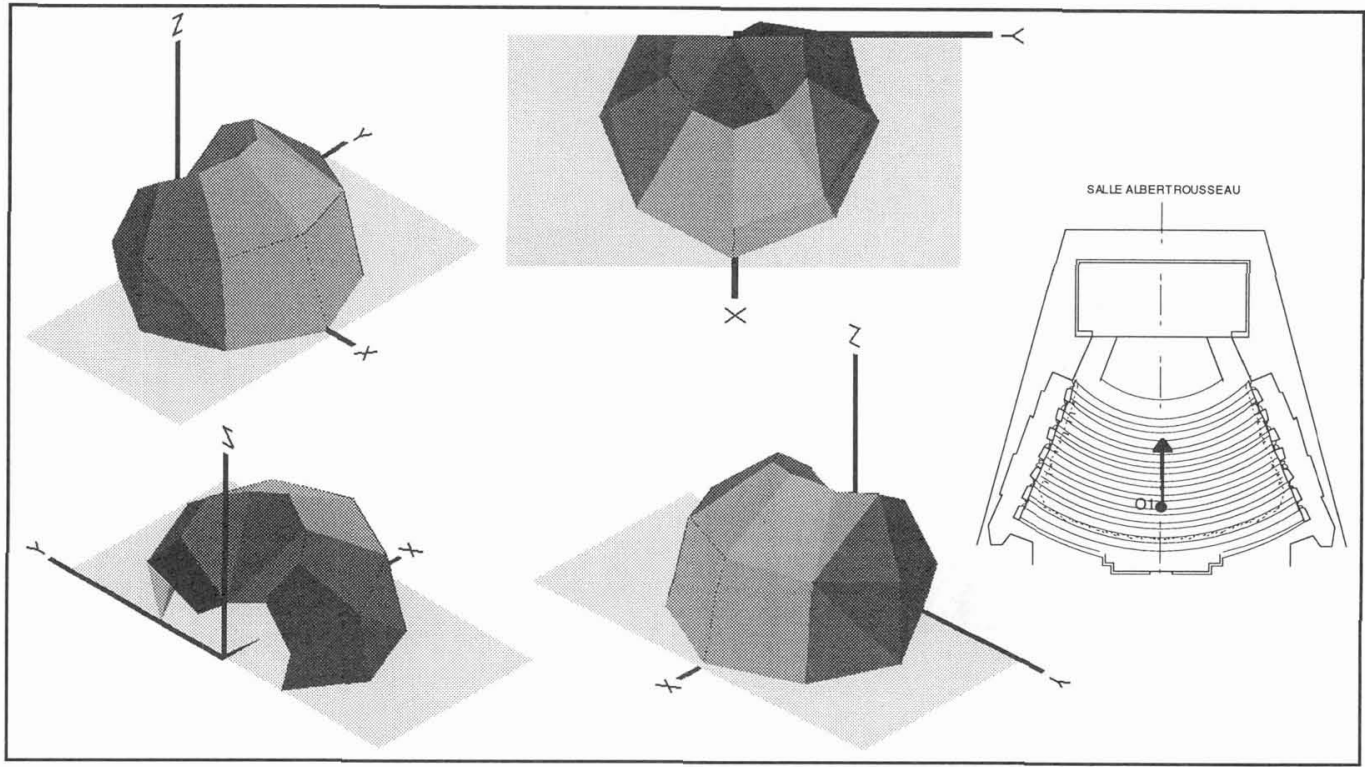

FIG. N23: Représentation vectorielle montrant la faiblesse des réflexions du plafond (salle Albert Rousseau).

Le premier exemple, pris dans la bande au tiers d'octave de $1 \mathrm{kHz}$, montre, dans le fond du parterre mais en avant des balcons, la proportion réduite des réflexions en provenance du plafond de la salle, alors que le son direct et les réflexions latérales dominent (Fig. n⿳3). Le second exemple, placé latéralement dans les rangées du milieu du parterre, met en évidence, dans la bande au tiers d'octave de $2 \mathrm{kHz}$, l'influence des réflexions latérales du côté "jardin", bien équilibrées cependant par le son direct, de même qu'une efficacité restreinte des réflecteurs du plafond, plutôt effectifs pour le balcon (Fig. n4).

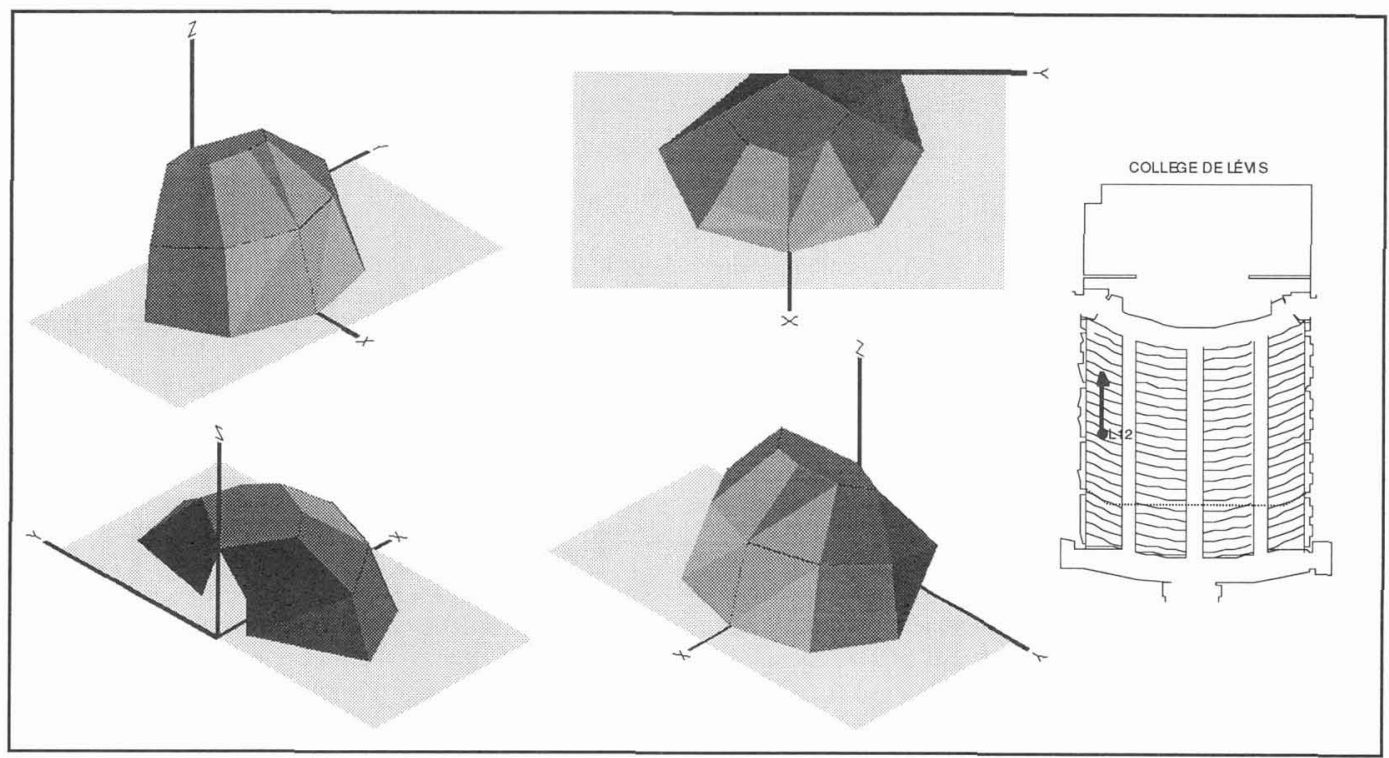

FIG. N4: Représentation vectorielle montrant l'influence des réflexions latérales (audittorium du collège de Lévis). 


\section{ANALYSE DE L'ÉQUILIBRE SPECTRAL DES RÉFLEXIONS}

Comme le montre finalement la figure $\mathrm{n}^{\mathrm{9}} 5$, il est possible d'exploiter ces résultats simultanément dans toutes les bandes de fréquence au tiers d'octave, en utilisant une représentation facile à interpréter, qui exprime tout autant la directivité des réflexions que les niveaux d'intensité. Pour le point déjà considéré dans l'axe du parterre, on peut constater la forte enveloppe sonore dans les basses fréquences, la directivité prononcée du son direct dans la bande de $4 \mathrm{kHz}$ et l'efficacité des réflexions latérales sur les murs des corbeilles à $2 \mathrm{kHz}$.

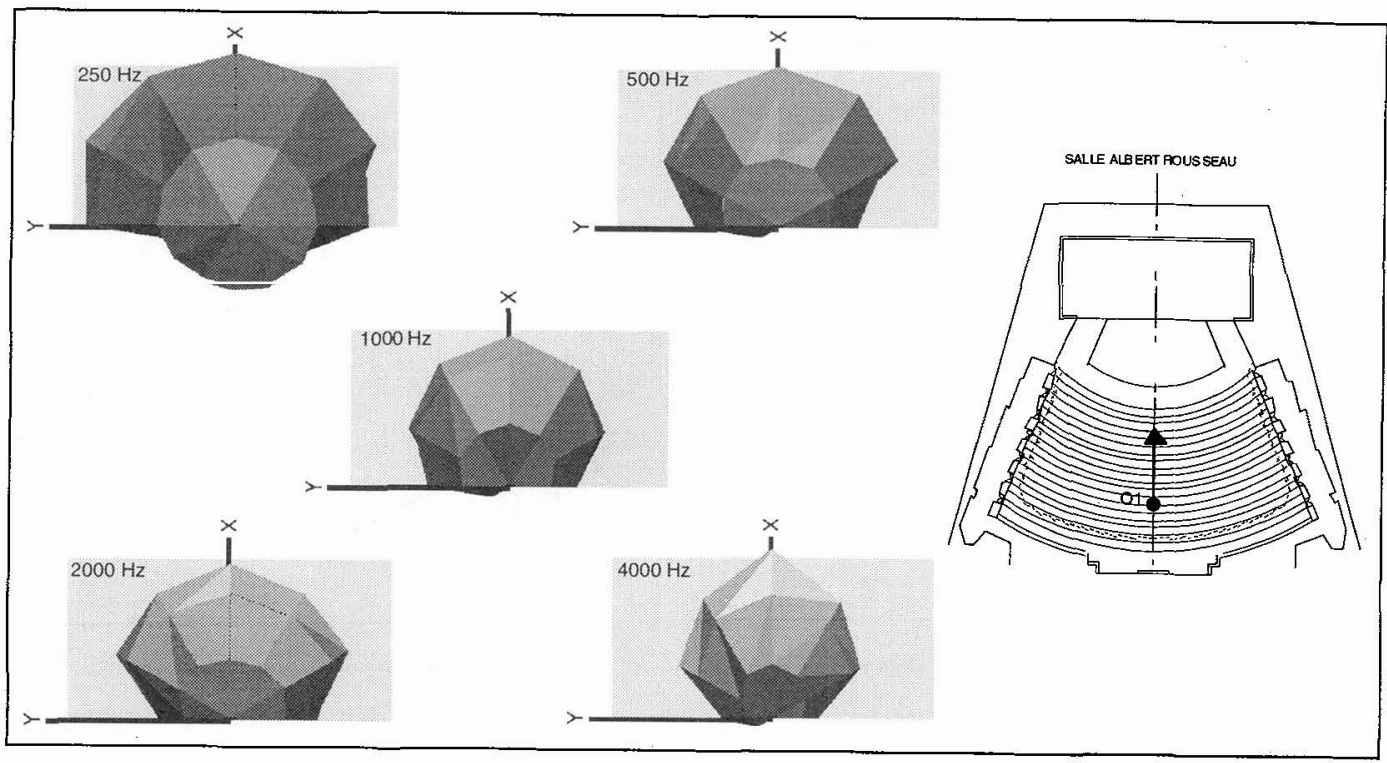

FIG. N5: Répartition de l'énergie dans les différentes bandes de fréquence (salle Albert Rousseau).

\section{CONCLUSION}

Les techniques intensimétriques présentées peuvent constituer un outil précieux pour l'analyse et le réglage fin des réflexions dans une salle de concert ou de spectacle, elles viennent compléter les procédures classiques de mesure de la réponse impulsionnelle ou de l'intelligibilité (avec des indices tels que EDT, C80 STI et G). D'autre part, elles permettent d'évaluer de façon précise les contributions des réflexions latérales dans les différentes bandes de fréquence, rejoignant ici des procédures telles que LF5 ("lateral energy fraction") [10].

\section{RÉFÉRENCES}

[1] FAHY, F.J., Sound Intensity, Elsevier Applied Science, 1987.

[2] MIGNERON J.-G.et ASSELINEAU M., "Utilisation de l'intensimétrie de part et d'autre d'un échantillon de façade soumis à l'impact du bruit de la circulation", pp. 53-61, in Revue d'Acoustique, № 80, Paris, octobre 1987.

[3] ABDOU, A. et GUY, R.W., "A PC based measurement system for obtaining spatial information and objective roomacoustic indicators", pp. 9-14, in Canadian Acoustics, Vol. 21, №1, 1993.

[4] PÉPIN, H.," Caractérisation du champ diffus dans les locaux par intensimétrie", pp.1085-1088, in Coll. de physique, Premier congr. français d'acoust., suppl. au N² 2 tome 51, février 1990.

[5] YAMASAKI, Y. et ITOW, T., "Measurement of spatial information in sound fields by closely located four point microphones method", pp.101-110, in Journ.Acoust. Soc. Jap., Vol. 10, N², 1989.

[6] MigNERON, J.-G., "Étude intensimétrique du champ sonore autour de différents postes de travail, modélisation de la diffraction et de la protection résultant d'enceintes partielles", pp.56-61, in Semaine Can. d'Acoust., Calgary, octobre 1987.

[7] RASMUSSEN, G., "Measurements of vector fields", pp.53-58, in 2nd Int. Congr. on Acoustic Intensity, CETIM, Senlis (France), 23 au 26 septembre 1985.

[8]MIGNERON J.-G., "Analyse acoustique du Grand Thêâtre de Québec avec utilisation de l'intensimétrie acoustique pour l'évaluation des réflecteurs" (avec la coll. de Woodcock, R. et Asselineau, M.), pp. 99-103, in Congr. Int. d'Acoust., Symp. de Vancouver, août 1986.

[9] MIGNERON J.-G. et LEMIEUX, P., "Mise an point d'une technique d'intensimétrie polaire: perspectives d'utilisation", pp. 457-460, in Journal de Physique IV, Vol. 2, Deux. Congr. Fr. d'Acoust., avril 1992.

[10] BRADLEY J.S., "The Evolution of Newer Auditorium Acoustics Measures", pp. 13-23, in Canadian Acoustics, Vol. 18, $\mathrm{N}^{2} 4,1992$. 